\title{
Morfometria dos canais e anéis inguinais de fetos natimortos e cadáveres adultos humanos e sua relação com as hérnias inguinais
}

\author{
Morphometric analysis of inguinal canals and rings of human fetus and adult \\ corpses and its relation with inguinal hernias
}

Carlos Manoel de Oliveira Figueiredo ${ }^{1}$; SÔnia Oliveira lima ${ }^{2}$; Sebastião Duarte Xavier Júnior ${ }^{3}$; Caroline Baptista da Silva ${ }^{4}$

\author{
R E S U M O
}

\begin{abstract}
Objetivo: Avaliar as diferenças anatômicas na região inguinal de fetos e adultos do gênero masculino, assim como a existência de uma possível base morfológica para a maior incidência de hérnias inguinais à direita em crianças e adultos. Métodos: Foram dissecados 20 fetos natimortos e 20 cadáveres humanos adultos in natura, todos do gênero masculino, comparando-se o comprimento do canal inguinal, o maior diâmetro dos anéis inguinais superficial e profundo em ambos os lados de cada cadáver e a existência ou não de superposição entre os anéis superficial e profundo. Resultados: Não foram observadas, nos dois grupos, diferenças significativas na comparação das medidas dos anéis profundos e superficiais, assim como dos canais inguinais, com seus respectivos contralaterais em cada espécime. Entretanto, houve diferença significativa entre os diâmetros dos anéis inguinais homolaterais, sendo o anel superficial maior do que o profundo nos fetos $(p=0,0002)$ e nos cadáveres adultos $(p<0,0001)$. A razão canal inguinal/altura mostrou diferença significativa entre os grupos $(p<0,0001)$, evidenciando que o canal inguinal em fetos é relativamente mais curto que em adultos. Foi observada, também, superposição dos anéis inguinais superficial e profundo ipsilaterais em dois fetos, porém não houve em nenhum dos adultos. Conclusão: A morfometria dos canais e anéis inguinais não justifica a maior incidência de hérnia do lado direito no gênero masculino.
\end{abstract}

Descritores: Canal Inguinal. Hérnia inguinal. Anatomia Regional. Feto.

\section{INTRODUÇÃO}

$A^{n}$ nualmente no mundo se operam 20.000 .000 de hérnias inguinais, representando grande parte das intervenções em cirurgia geral ${ }^{1}$. Esse tipo de alteração acomete mais frequentemente o gênero masculino em todas as faixas etárias, sendo mais comum do lado direito ${ }^{2-4}$. Apesar de haver várias técnicas descritas para o tratamento dessa doença ${ }^{5}$, ela ainda representa um desafio cirúrgico permanente ${ }^{6,7}$

A incidência de hérnia inguinal congênita atinge cerca de $1 \%$ a $3 \%$ da população geral. Ela é considerada a condição cirúrgica mais frequente em crianças de todas as idades, surgindo mais comumente como o tipo indireto ${ }^{7-}$ ${ }^{10}$. Seu reparo é um dos procedimentos mais comumente realizados pelo cirurgião pediátrico ${ }^{11}$.

No presente estudo, foi avaliada a medida das estruturas anatômicas (morfometria) dos canais e anéis inguinais de fetos natimortos e cadáveres adultos humanos do gênero masculino, comparando os resultados de ambos os grupos entre si. Objetivou-se investigar a possível base anatômica para a maior incidência das hérnias inguinais indiretas à direita em crianças e adultos do gênero masculino, assim como correlacionar com as técnicas cirúrgicas via inguinotomia utilizadas para tratamento dessas hérnias em ambos os grupos.

\section{MÉTODOS}

Foram utilizados 20 fetos humanos natimortos conservados em formol a 10\% (obtidos dos laboratórios de Anatomia Humana da Universidade Federal de Sergipe e da Universidade Tiradentes/ Aracaju - SE), com idade gestacional maior que 30 semanas e 20 cadáveres adultos humanos in natura (obtidos do Instituto Médico Legal de Sergipe). Os grupos eram do gênero masculino e não apresentavam doenças na região inguinal.

Nos dois grupos, foram registrados comprimento (altura) e idade. Nos fetos, a idade gestacional foi estimada pelo método somático de Capurro' ${ }^{2}$. Nos adultos, a idade foi registrada pela análise do documento de identidade ou, na falta deste, pela estimativa realizada pelo médico legista.

Trabalho realizado nos Laboratórios de Anatomia Humana da Universidade Federal de Sergipe e da Universidade Tiradentes (SE), e no Instituto Médico Legal de Sergipe - SAE-BR.

1. Acadêmico de Medicina da Universidade Federal de Sergipe - SE-BR; 2. PhD, Coordenadora da disciplina de Clínica Cirúrgica do Departamento de Medicina da Universidade Federal de Sergipe - SE-BR; 3. Cirurgião Pediátrico do Hospital Universitário de Sergipe-SE-BR; 4. Acadêmica de Medicina da Universidade Federal de Sergipe- SE-BR. 
Na dissecção, foram medidos, com auxílio de lupa de $5 x$ e paquímetro, o comprimento do canal inguinal, o maior diâmetro do anel inguinal superficial e do anel inguinal profundo em ambos os lados de cada cadáver. Foi considerado o comprimento do canal inguinal como a distância entre os anéis inguinais superficial e profundo. Foi observada a existência ou não de superposição entre os anéis superficial e profundo de cada lado de todos os espécimes. Considerou-se superposição quando a distância entre os eixos verticais (paralelos à linha mediana) dos anéis era igual ou inferior a um milímetro.

Foram calculados média e desvio-padrão para as medidas de cada estrutura e os resultados foram avaliados estatisticamente pelo teste " $T$ " (Student), com nível de significância $p<0,05$. Os anéis inguinais foram comparados unilateralmente (anel superficial com o profundo) e bilateralmente (cada anel com seu homólogo contralateral) em todos os espécimes estudados. O canal inguinal foi comparado com seu contralateral e foi calculada a razão: média do comprimento dos canais inguinais (direito e esquerdo)/ altura de cada espécime. Os resultados dessa relação foram comparados entre os dois grupos.

O presente estudo foi aprovado pelo Comitê de Ética em Pesquisa da Universidade Federal de Sergipe, com protocolo de $n^{\circ}$ 072/2003.

\section{RESULTADOS}

Os fetos natimortos (Grupo 1) apresentaram uma idade gestacional média de 36,9 semanas (variando de 30,6 a 42,6 semanas) e um comprimento médio de 42,5 centímetros (variando de $30 \mathrm{~cm}$ a $55,5 \mathrm{~cm}$ ). Nos cadáveres adultos (Grupo 2), a idade variou de 18 anos a 68 anos (média de 35,1 anos) e o comprimento (altura), de 1,60m a 1,78m (média de 1,70m).

Nos dois grupos, não foram observadas diferenças significativas na comparação das medidas dos anéis profundos e superficiais, assim como dos canais inguinais, com seus respectivos contralaterais em cada espécime. Entretanto, houve diferença significativa entre os diâmetros dos anéis inguinais homolaterais, sendo 0 anel superficial mais largo do que o profundo nos fetos $(p=0,0002)$ e nos cadáveres adultos $(p<0,0001)$ (Tabelas 1 e 2).

Observou-se superposição dos anéis inguinais superficial e profundo ipsilaterais em dois fetos (7,5\%). Em um deles, a superposição foi bilateral, e no outro ocorreu apenas do lado direito. Nenhum dos cadáveres adultos apresentou tal superposição.

A razão canal inguinal/altura mostrou diferença significativa entre os grupos 1 e 2 ( $p<0,0001)$, evidenciando que o canal inguinal em fetos é relativamente mais curto que em adultos.

\section{DISCUSSÃO}

Em crianças, apesar de a hérnia inguinal se dar, geralmente, por persistência do conduto peritônio-vaginal ${ }^{2}$, a permanência dessa estrutura não implica obrigatoriamente em ocorrência de hérnia inguinal congênita ${ }^{13}$. A maior freqüência dessa doença no lado direito poderia ter como uma de suas justificativas a existência de diferenças nas dimensões de canais e anéis inguinais entre os dois lados. Embora não tenham sido encontradas diferenças significativas na morfometria dessas estruturas, algumas particularidades anatômicas dos fetos em relação aos cadáveres adultos foram encontradas. Verificou-se, nos primeiros, um comprimento mais curto dos canais inguinais, além de superposição dos anéis inguinais externo e interno ipsilaterais, ainda que com baixa freqüência (7,5\%). De acordo com Tanyel et al. ${ }^{14}$, o canal inguinal permanece curto até os dois anos de idade, quando passa a ter crescimento linear. Essas características dão substrato para correção de hérnias inguinais em crianças por inguinotomia

Tabela 1 - Medida (em centímetros) do canal inguinal, anéis inguinais superficiais e profundos de fetos natimortos.

\begin{tabular}{lccc}
\hline Estrutura & Lados direito e esquerdo $M \pm D P$ & Lado direito $M \pm D P$ & Lado esquerdo $M \pm D P$ \\
\hline Canal Inguinal & $0,708 \pm 0,0374$ & $0,698 \pm 0,055$ & $0,718 \pm 0,0513$ \\
Anéis inguinais superficiais & $0,360 \pm 0,0141$ & $0,365 \pm 0,00199$ & $0,355 \pm 0,0205$ \\
Anéis inguinais profundos & $0,283 \pm 0,0140$ & $0,298 \pm 0,0190$ & $0,268 \pm 0,0206$ \\
\hline
\end{tabular}

$M \pm D P:$ Média \pm desvio-padrão da média.

Tabela 2 - Medida (em centímetros) do canal inguinal, anéis inguinais superficiais e profundos de cadáveres adultos.

\begin{tabular}{lccc}
\hline Estrutura & Lados direito e esquerdo $\mathbf{M} \pm \mathrm{DP}$ & Lado direito $\mathbf{M} \pm \mathbf{D P}$ & Lado esquerdo $\mathbf{M} \pm \mathrm{DP}$ \\
\hline Canal Inguinal & $4,34 \pm 0,994$ & $4,35 \pm 0,1520$ & $4,32 \pm 0,1320$ \\
Anéis inguinais superficiais & $1,23 \pm 0,0563$ & $1,23 \pm 0,0563$ & $1,19 \pm 0,0621$ \\
Anéis inguinais profundos & $0,83 \pm 0,0238$ & $0,81 \pm 0,0285$ & $0,85 \pm 0,0384$ \\
\hline
\end{tabular}

M DP: Média \pm desvio-padrão da média. 
sem a abertura da aponeurose do músculo oblíquo externo ${ }^{15}$. Em adultos, a abertura dessa aponeurose é etapa obrigatória na correção cirúrgica de hérnias por inguinotomia ${ }^{16}$.

Nos dois grupos, o anel superficial mostrou-se mais largo do que o anel profundo homolateral tanto à direita quanto à esquerda. Contudo, o canal inguinal e seus anéis não diferem em relação aos contralaterais no mesmo espécime. A morfometria dos canais e anéis inguinais não justifica, salvo pesquisas mais profundas, a maior incidência de hérnia do lado direito no gênero masculino.

\section{Agradecimento}

Agradecemos ao Prof. Dr. Francisco do Prado Reis, coordenador do Laboratório de Anatomia Humana da Universidade Tiradentes - SE, pela autorização da utilização dos fetos natimortos empregados nesta pesquisa.

\title{
A $B$ S S T R R A C T
}

\begin{abstract}
Objective: To evaluate the anatomical differences of fetus and adults concerning the inguinal region of male gender, as well as the presence of possible morphological basis for the higher incidence of inguinal hernias in the right side in both groups. Methods: Twenty human stillborn fetuses and twenty in natura adult male corpses were dissected in order to compare the length of the inguinal canals, the longer diameter of the deep and superficial inguinal rings in both sides of each corpse, and the presence or not of overlap between the deep and superficial rings. Results: No statistically significant differences were observed in both groups regarding the measures of the deep and the superficial rings, as well as the inguinal canals, when in comparison to the respective contralateral anatomical structures of each specimen. Nevertheless, there was a statistically significant difference between the diameter of homolateral inguinal rings, once the superficial ring was longer than the deep ring in fetus $(p=0.0002)$ and adult corpses $(p<0.0001)$. The ratio inguinal canal/height showed to be statistically different between both groups $(p<0.0001)$, since the inguinal canal in fetus is shorter than in adults. It was also observed overlapping of homolateral superficial and deeper inguinal rings in two fetuses, but none in adults. Conclusion: Morphometric analysis of the inguinal canals and rings does not justify the higher incidence of hernias in the right side in the male gender.
\end{abstract}

Key words: Inguinal canal. Inguinal hernia. Regional anatomy. Fetus.

\section{REFERENCIAS}

1. Venturelli MF, Uherek FP, Cifuentes CV, Folch PC, Felmer OE, Valentin PH. Hernia inguinal: conceptos actuales. Cuad Cir. 2007; 21(1):43-51

2. Towsend CM, Beauchamp RD, Evers BM, Mattox KL, editors. Sabiston - Tratado de cirurgia: as bases biológicas da prática cirúrgica moderna. Rio de Janeiro: Guanabara Koogan; 2003.

3. Almeida M, Sousa AA, Alves MR, Giroldo SM, Otta EK, Bernatt J et al. Hérnia inguinal congênita: um estudo prospectivo. Rev Med Paraná. 2000; 58(1):50-62

4. Minossi JG. Cirurgia pediátrica. Rio de Janeiro: Revinter; 2003.

5. Nagem RG, Silva AL. Comparação entre as técnicas de Shouldice modificada por Berliner e a de Falci-Lichtenstein na hernioplastia inguinal. Rev Col Bras Cir. 2007; 34(5):331-5.

6. Gac PE, Uherek FP, Del Pozo ML, Oropesa AVC, Rocco ER. Hernia inguinal: un desafío quirúrgico permanente. Cuad Cir. 2001; 15(1):96-106

7. Trindade MRM. Hérnia inguinal. Einstein (São Paulo). 2005; 3(2):140.

8. Cox JA. Inguinal hernia of childhood. Surg Clin North Am. 1985; 65(5):1331-42

9. Richham PP, Soper RT, Stauffer UG et al. Synopsis of pediatric surgery. Chicago: Year Book Medical Publishers; 1975.

10. Raia AA, Zerbini EJ, coord. Clínica cirúrgica Alípio Correia Netto. São Paulo: Sarvier; 1994

11. Raffenspenger JG. Swenson's pediatric surgery. New York: Appleton-Century-Crofts; 1980.
12. Segre CAM, Armellini PA, Marino WT. RN. $5^{\text {a }}$ ed. São Paulo: Sarvier; 1995.

13. Peristein J, Du Bois JJ. The role of laparoscopy in the management of suspected recurrent pediatric hernias. J Pediatrics Surg. 2000; 35(8):1205-8.

14. Tanyel FC, Ocal T, Karaagaoglu E, Büyükpamukçu N. Individual and associated effects of length of inguinal canal and caliber of the sac on clinical outcome in children. J Pediatr Surg. 2000; 35(8):1165-9.

15. Macksoud JG, editor. Cirurgia pediátrica. $2^{\text {a }}$ ed. Rio de Janeiro: Revinter, 2003.

16. Vieira OM, Chaves $C P$, Manso JEF, Eulálio JMR. Clínica cirúrgica: fundamentos teóricos e práticos. São Paulo: Atheneu; 2004.

Recebido em 06/11/2008

Aceito para publicação em 15/01/2009

Conflito de interesse: nenhum

Fonte de financiamento: nenhuma

\section{Como citar este artigo:}

Figueiredo CMO, Lima SO, Xavier Júnior SD, Silva CB. Morfometria dos canais e anéis inguinais de fetos natimortos e cadáveres adultos humanos e sua relação com as hérnias inguinais. Rev Col Bras Cir. [periódico na Internet] 2009; 36(4). Disponível em URL: http://www.scielo.br/rcbc

\section{Endereço para correspondência:}

Sônia Oliveira Lima

E-mail: sonia.sol@ ibest.com.br 